\title{
Current neurosurgical indications for saphenous vein graft bypass
}

\author{
Jonathan A. Friedman, M.D., And David G. Piepgras, M.D. \\ Department of Neurologic Surgery, Mayo Clinic, Rochester, Minnesota
}

\begin{abstract}
Object. Vascular bypass is performed in neurosurgery for a variety of pathological entities, including extracranial atherosclerotic disease, extra- and intracranial aneurysms, and tumors involving the carotid artery (CA) at the skull base or cervical regions. Creation of an interposition saphenous vein graft (SVG) is the typical method of choice when the superficial temporal artery is not an option.

Methods. One hundred thirty consecutive patients treated with SVG between July 1988 and December 2002 at the Mayo Clinic were studied. A total of 130 procedures were performed in 130 patients. The indications were intracranial aneurysm in 51 patients (39\%), CA occlusive disease in $36(28 \%)$, extracranial CA aneurysm in 17 (13\%), tumors involving the cervical CA in $11(8 \%)$, vertebral artery occlusive disease in eight $(6 \%)$, and other indications in six patients (5\%). Among patients treated for intracranial aneurysms, 43 harbored giant aneurysms $(>25 \mathrm{~mm}$ in widest diameter) whereas the remaining eight patients harbored aneurysms that were large (15-25 $\mathrm{mm}$ in widest diameter). Among patients with CA occlusive disease, high-grade stenosis at the CA bifurcation was present in 29 and CA occlusion was demonstrated in seven.

Conclusions. The use of SVG bypass remains a valuable component of the neurosurgical armamentarium for a variety of pathological entities. Despite a general trend toward decreased use because of improved endovascular technology, surgical facility with this procedure should be maintained.
\end{abstract}

\section{KEY WORDS • saphenous vein graft • revascularization • carotid artery • occlusion • cerebral aneurysm • skull base tumor}

Saphenous vein graft bypass has been a valuable component of the neurosurgical armamentarium for a relatively wide spectrum of pathological entities. The indications for interposition SVG bypass continue to evolve as technical experience is gained and disseminated and as alternative therapies are introduced. In this study, we report the frequency of SVG bypass for neurosurgical pathological lesions at our institution over the past 14 years and discuss trends and current indications for its use.

\section{CLINICAL MATERIAL AND METHODS}

One hundred thirty consecutive patients underwent SVG bypass between September 1988 and December 2002 at Mayo Clinic, in Rochester, Minnesota. The number of patients who underwent surgery annually is shown in Fig. 1. The procedures were performed using standard techniques for SVG bypass that have been described previously. ${ }^{5-7,9}$ All bypass procedures, whether planned preoperatively

\footnotetext{
Abbreviations used in this paper: $\mathrm{BA}=$ basilar artery; $\mathrm{BTO}=$ balloon test occlusion; $\mathrm{CA}=$ carotid artery; $\mathrm{CEA}=$ carotid endarterectomy; ICA = internal CA; MCA = middle cerebral artery; STA = superficial temporal artery; $\mathrm{SVG}=$ saphenous vein graft; $\mathrm{VA}=$ vertebral artery; VBJ = vertebrobasilar junction.
}

or performed based on unexpected intraoperative findings, were studied. Three patients $(2 \%)$ underwent a second SVG bypass to revise an occluded vein graft postoperatively, and these second procedures were not included in the analysis.

\section{RESULTS}

One hundred thirty procedures were performed in 130 patients. The indications were intracranial aneurysm in 51 patients $(39 \%)$, CA occlusive disease in $36(28 \%)$, extracranial CA aneurysm in $17(13 \%)$, tumors involving the cervical CA in $11(8 \%)$, VA occlusive disease in eight $(6 \%)$, and other indications in six (5\%) (Table 1).

Among patients treated for intracranial aneurysms, 43 harbored giant aneurysms ( $>25 \mathrm{~mm}$ in widest diameter) whereas eight harbored large aneurysms $(15-25 \mathrm{~mm}$ in widest diameter). The aneurysms were located on the ICA in 42 cases, the MCA in four cases, the BA trunk or apex in three cases, and the VBJ in two cases. In general, the SVG bypass was constructed in preparation for concomitant or delayed ipsilateral parent vessel occlusion for treatment of an aneurysm that could not be repaired directly.

Among patients with CA occlusive disease, high-grade stenosis at the CA bifurcation was present in 29 and CA 


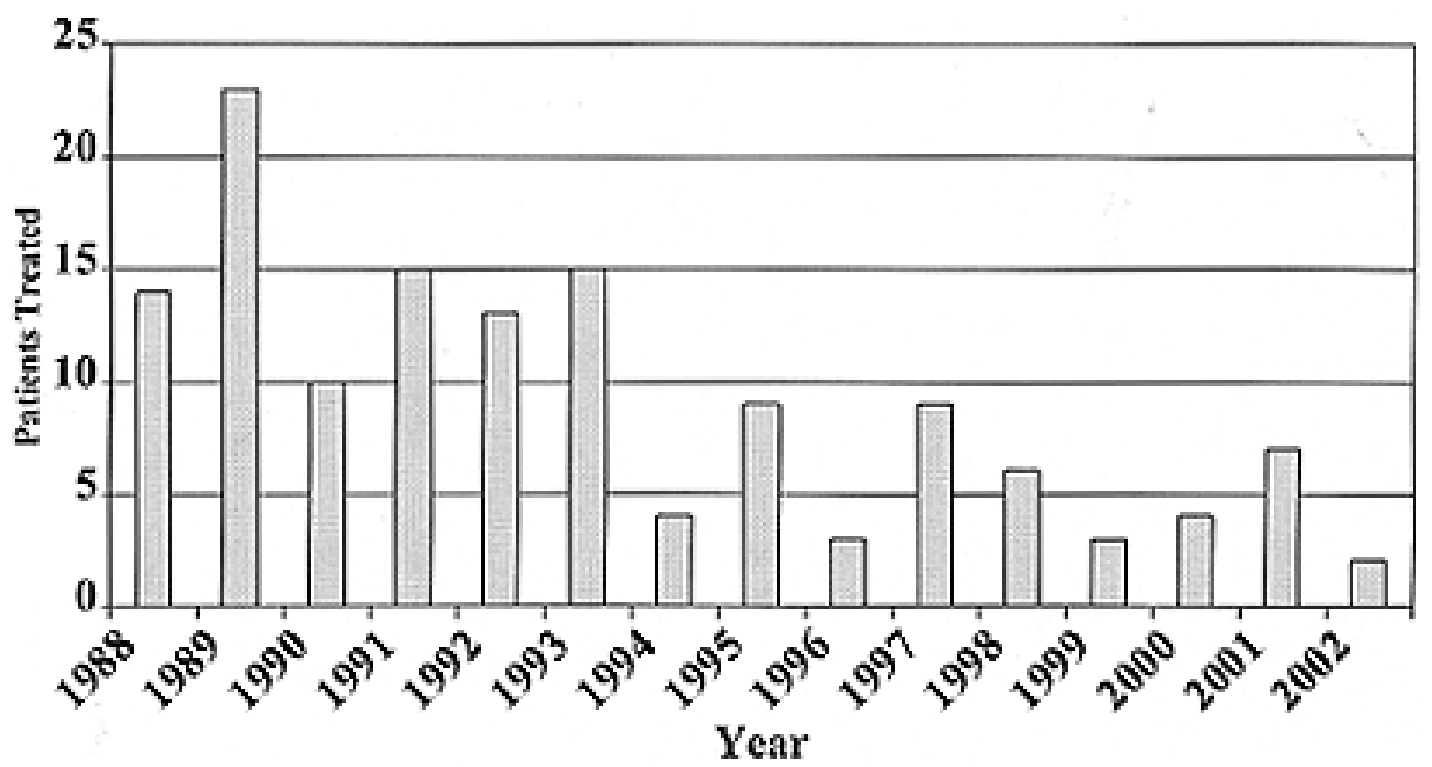

Fig. 1. Bar graft demonstrating the number of patients treated annually; the 1988 and 2002 totals are projected for a full year.

occlusion was present in seven. All patients experienced preoperative symptoms of cerebral ischemia or infarction. Among patients with high-grade CA stenosis, 22 had undergone prior CEA, whereas seven required SVG bypass at the initial surgery. In general, bypass for CA occlusion was reserved for a selected subset of symptomatic patients in whom STA-MCA bypass was technically not feasible. Among patients with VA occlusive disease, high-grade stenosis was demonstrated in four and VA occlusion in four; all patients were symptomatic.

The other indications for SVG bypass included cervical CA dissection without aneurysm in two patients, CA cavernous fistula in one patient, reconstruction of the external CA system to allow access to embolize a facial arteriovenous malformation in one patient, proximal high-grade MCA stenosis in one patient, and moyamoya disease in one patient.

\section{DISCUSSION}

\section{Intracranial Aneurysms}

Saphenous vein bypass grafting has proven invaluable for the treatment of intracranial aneurysms not amenable to direct repair, ${ }^{4-6}$ and this was the most frequent indication for bypass in our experience. The vast majority of aneurysms treated in this way were larger than $25 \mathrm{~mm}$ in diameter, and the majority were intracavernous ICA aneurysms. The bypass was generally accompanied by trapping or parent vessel sacrifice for treatment of the aneurysm. In patients in whom preoperative BTO was tolerated, bypass was generally not performed. In patients in whom this is not tolerated or have not undergone BTO, or in whom there are other contraindications to CA sacrifice such as contralateral CA aneurysm, SVG bypass was typically performed. Although this strategy was planned preoperatively in most cases, in some patients it became evident only intraoperatively that the lesion would not be satisfactorily repaired using direct clip reconstruction while maintaining patency of the parent vessel. It is advisable that in approaching a large, complex intracranial aneurysm for which clipping may not be feasible or primary trunk occlusion may be required that the surgeon be prepared for and capable of performing SVG bypass if necessary. Although most vein bypass grafts have been used for anterior circulation lesions, posterior circulation bypass in cases involving giant aneurysms of the BA or VBJ was occasionally required, usually into the posterior cerebral artery.

\section{Extracranial Arterial Occlusion}

For the primary treatment of high-grade CA stenosis, CEA is the procedure of choice and is not possible only in rare cases, such that SVG bypass involving reconstruction of the ICA is necessary. In our 14-year experience involving this series, this was necessary in only seven cases. These were unusual circumstances including a highly diseased, redundant $\mathrm{CA}$ due to underlying fibromuscular

TABLE 1

Indications for SVG bypass

\begin{tabular}{lc}
\hline \hline Indication & No. of Cases \\
\hline intracranial aneurysm & 51 \\
ICA & 42 \\
MCA & 4 \\
BA trunk & 3 \\
VBJ & 2 \\
CA stenosis & 29 \\
primary & 7 \\
reoperation & 22 \\
CA occlusion & 7 \\
VA stenosis & 4 \\
VA occlustion & 4 \\
cervical CA tumor & 11 \\
extracranial CA aneurysm & 17 \\
other & 6 \\
\hline
\end{tabular}


dysplasia, stenosis extending very distally in the cervical $\mathrm{CA}$, and tandem stenosis of the CA siphon necessitating extracranial-intracranial bypass. Recurrent CA atherosclerosis and stenosis following previous CEA was a more common indication for a extracranial interpositional SVG. In these cases, the recurrent disease was so extensive that repair of the artery with repeated CEA and patch graft was not possible. As experience and technology involving CA angioplasty and stent placement improves, this may become a more commonly performed treatment for recurrent CA stenosis, but the efficacy and morbidity associated with angioplasty and stent placement in this setting has yet to be thoroughly established.

Our use of extracranial-intracranial bypass for the treatment of cerebral ischemia due to CA occlusion diminished substantially when the efficacy of the procedure was not demonstrated in a prospective randomized trial. ${ }^{2}$ Furthermore, we generally prefer performing STA-MCA bypass instead of SVG bypass in the setting of chronic ischemia, because the high-flow vein graft is associated with higher risks, including acute graft thrombosis, and particularly hemorrhagic complications due to hyperperfusion. ${ }^{8}$ Primarily, SVG bypass was undertaken in this setting when the STA was not suitable for use as a donor vessel.

Saphenous vein bypass for vertebrobasilar occlusive disease was more frequently conducted early in this series. Without question, endovascular techniques have improved, supplanting bypass as a first-line therapy for this indication, $, 1,3$ as evidenced by the fact that the last SVG bypass for VA occlusive disease performed at our institution was in 1995, and all but one were performed prior to 1993. Nevertheless, bypass of the posterior circulation is occasionally necessary (see Intracranial Aneurysms) and collective skills should therefore be maintained.

\section{Tumors and Other Indications}

Extracranial SVG bypass was conducted for rare cervical tumors involving the CA, particularly in patients with a history of cervical resection and radiotherapy. Surprisingly, we did not perform SVG bypass in cases involving skull base or intracranial tumors during the study period. Although this may suggest underutilization of the bypass procedure in this setting, the indications and benefit for radical resections of many skull base lesions remains poorly defined. Rare indications for SVG bypass, such as CA "blowout" or special vascular access for endovascular procedures, will continue to occur and require appropriate treatment.

\section{Cerebral Revascularization Trends}

Clearly, at our institution, the use of SVG bypass in neurosurgery has decreased over the past 14 years (Fig. 1), largely because of improved endovascular therapies as well as more reliable information for tolerance to primary trunk occlusion as ascertained by preoperative BTO studies. Over the latter half of the study period, however, the number of procedures performed annually has not progressively decreased, indicating that a small but consistent number of SVG bypass procedures will continue to be required in the treatment of complex cerebrovascular disease. In this light, it will likely be an ongoing challenge for cerebrovascular neurosurgeons to maintain the confidence and expertise necessary to perform this technically demanding procedure.

\section{References}

1. Barakate MS, Snook KL, Harrington TJ, et al: Angioplasty and stenting in the posterior cerebral circulation. J Endovasc Ther 8:558-565, 2001

2. EC/IC Bypass Study Group: Failure of extracranial-intracranial arterial bypass to reduce the risk of ischemic stroke. Results of an international randomized trial. N Engl J Med 313: 1191-1200, 1985

3. Levy EI, Horowitz MB, Koebbe CJ, et al: Transluminal stentassisted angioplasty of the intracranial vertebrobasilar system for medically refractory, posterior circulation ischemia: early results. Neurosurgery 48:1215-1221, 2001

4. Santoro A, Guidetti G, Dazzi M, et al: Long saphenous-vein grafts for extracranial and intracranial internal carotid aneurysms amenable neither to clipping nor to endovascular treatment. J Neurosurg Sci 43:237-250, 1999

5. Sundt TM Jr, Piepgras DG, Houser OW, et al: Interposition saphenous vein grafts for advanced occlusive disease and large aneurysms in the posterior circulation. J Neurosurg 56: 205-215, 1982

6. Sundt TM Jr, Piepgras DG, Marsh WR, et al: Saphenous vein bypass grafts for giant aneurysms and intracranial occlusive disease. J Neurosurg 65:439-450, 1986

7. Sundt TM Jr, Piepgras DG, Meyer FB, et al: Vein bypass grafts for giant aneurysms and severe intracranial occlusive disease in the anterior and posterior circulation, in FB Meyer (ed): Sundt's Occlusive Cerebrovascular Disease, ed 2. Philadelphia: WB Saunders, 1994, pp 489-511

8. Sundt TM Jr, Whisnant JP, Fode NC, et al: Results, complications, and follow-up of 415 bypass operations for occlusive disease of the carotid system. Mayo Clin Proc 60:230-240, 1985

9. Sundt TM III, Sundt TM Jr: Principles of preparation of vein bypass grafts to maximize patency. J Neurosurg 66:172-180, 1987

\section{Manuscript received January 31, 2003.}

Accepted in final form February 7, 2003.

Address reprint requests to: Jonathan A. Friedman, M.D., Department of Neurologic Surgery, Joseph 1-229, Saint Mary's Hospital, 1216 Second Street South West, Rochester, Minnesota 55905. email: friedman.jonathan@mayo.edu. 